DEMOGRAPHIC RESEARCH

VOLUME 35, ARTICLE 18, PAGES 505-534

PUBLISHED 26 AUGUST 2016

http://www.demographic-research.org/Volumes/Vol35/18/

DOI: 10.4054/DemRes.2016.35.18

Research Article

Fertility progression in Germany:

An analysis using flexible nonparametric cure survival models

Vincent Bremhorst

Michaela Kreyenfeld

Philippe Lambert

(C)2016 Bremhorst, Kreyenfeld \& Lambert.

This open-access work is published under the terms of the Creative Commons

Attribution NonCommercial License 2.0 Germany, which permits use,

reproduction \& distribution in any medium for non-commercial purposes, provided the original author(s) and source are given credit.

See http:// creativecommons.org/licenses/by-nc/2.0/de/ 


\section{Contents}

$\begin{array}{lll}1 & \text { Introduction } & 506\end{array}$

2 Context and previous research 508

$3 \quad$ Methods and data 510

$3.1 \quad$ Methods and research strategy 510

3.1.1 Cure survival models 510

3.1.2 Bayesian inference 512

3.2 Data 513

3.2.1 Sample 513

3.2.2 Dependent variable: transition to a second and a third child 514

3.2.3 Independent variables $\quad 515$

$4 \quad$ Results $\quad 517$

4.1 Second births $\quad 517$

4.2 Third births 523

$\begin{array}{ll}4.3 & \text { Comparison with a Cox model } \\ \end{array}$

$\begin{array}{lll}5 & \text { Discussion } & 527\end{array}$

6 Acknowledgements $\quad 529$

References $\quad 530$ 


\title{
Fertility progression in Germany: An analysis using flexible nonparametric cure survival models
}

\author{
Vincent Bremhorst ${ }^{1}$ \\ Michaela Kreyenfeld ${ }^{2,3}$ \\ Philippe Lambert ${ }^{1,4}$
}

\begin{abstract}
OBJECTIVE

This paper uses data from the German Socio-Economic Panel (GSOEP) to study the transition to second and third births. In particular, we seek to distinguish the factors that determine the timing of fertility from the factors that influence ultimate parity progression.
\end{abstract}

\section{METHODS}

We employ cure survival models, a technique commonly used in epidemiological studies and in the statistical literature but only rarely applied to fertility research.

\section{RESULTS}

We find that education has a different impact on the timing and the ultimate probability of having a second and a third birth. Furthermore, we show that the shape of the fertility schedule for the total population differs from that of 'susceptible women' (i.e., those who have a second or a third child).

\section{CONCLUSION}

Standard event history models conflate timing and quantum effects. Our approach overcomes this shortcoming. It estimates separate parameters for the hazard rate of having a next child for the 'susceptible population' and the ultimate probability of having another child for the entire population at risk.

\footnotetext{
${ }^{1}$ Université catholique de Louvain, Institut de Statistique, Biostatistique et Sciences Actuarielles, Voie du Roman Pays 20, B-1348 Louvain-la-Neuve, Belgium. E-Mail: vincent.bremhorst@uclouvain.be.

${ }^{2}$ Hertie School of Governance GmbH Friedrichstraße 180, 10117 Berlin, Germany.

${ }^{3}$ Max Planck Institute for Demographic Research, Konrad-Zuse-Straße 1, 18057 Rostock, Germany.

${ }^{4}$ Université de Liège, Faculté des sciences sociales, Méthodes quantitatives en sciences sociales, Place des orateurs, 3 - Quartier Agora (Bat. B31), B-4000 Liège, Belgium.
} 


\section{CONTRIBUTION}

We go beyond standard cure survival models, also known as split population models, used in fertility research by specifying a flexible non-parametric model using Bayesian P-splines for the latent distribution (related to the timing of an extra birth) instead of a parametric model. Our approach is, so far, limited to time-constant covariates, but can be extended to include time-varying covariates as well.

\section{Introduction}

Event history models have a long tradition in demographic research. They represent a bridge between the classical life table methods and modern regression techniques (Hoem 1993). Unlike OLS regression, they are able to account for censoring and allow for a flexible specification of the baseline intensity and the integration of time-varying covariates into the analysis (Rizopoulos 2012). In fertility research, the most commonly used models are proportional hazard specifications, such as the piecewise exponential or the Cox model. Although they are widely used, these models have a serious shortcoming: They are unable to separate the impact of the covariates on the timing of births from the factors that influence the ultimate parity progression. As they cannot differentiate between timing and quantum, they often produce misleading results.

An example of this shortcoming is that models which try to unravel the impact of female education on second and third birth progressions tend to generate confusing estimates. For western European countries, these models have often shown that highly educated women have relatively high second and third birth rates (Berinde 1999; Kravdal 2001; Kreyenfeld 2002; Oláh 2003; Prskawetz and Zagaglia 2005; Gerster et al. 2007). There is, however, considerable ambivalence among demographers about how these results should be interpreted. A positive coefficient for a high level of education may indicate that highly educated women are more likely than less educated women to have a second or a third child. This is plausible, as a highly educated woman (or her partner) may be expected to have the earning power to afford a larger family. However, the results may also be indicative of differences in the timing of births among women of different educational levels. This is also a very plausible interpretation. As Ní Bhrolcháin (1986) has suggested, a highly educated woman may space her births relatively closely together to minimize interruptions of her employment career. This 'work-accelerated childbearing' leads to narrow birth intervals among the highly educated, but these women do not necessarily have a greater likelihood of progressing to births of higher order. While this may be the case, conventional event history models are unable to provide an unambiguous answer to the question of whether highly 
educated women are more likely to progress to higher order births, or whether they simply space their births closer together than other women. Our paper addresses the call for more "real-world justifications" (Ní Bhrolcháin 2011: 850) that show that it is worthwhile to separate the level and timing of fertility.

Data for this analysis come from the German-Socio-Economic Panel for the years 1984-2013 (version 31.0). Event history models have shown that in Germany, as in most other western European countries, women with higher levels of education have elevated second and third birth rates (Huinink 1989; Kreyenfeld 2002). In this paper, we try to cast new light on these findings by employing cure survival models - and, more precisely, promotion time models - in the analysis of second- and third-birth fertility. Cure survival models are increasingly used in the statistical literature and in epidemiological studies. However, as Alter, Oris, and Tyurin (2007) have pointed out, these families of models are, surprisingly, rarely used in fertility studies. One of the first studies that applied cure survival models in the realm of fertility research was performed by Yamaguchi and Ferguson (1995), who studied the role of education in the transition to second and third births in the US. Their results suggested that the probability of having a second child was significantly greater for highly educated women than for less educated women, but that less educated women spaced their births closer together than highly educated women. Furthermore, highly educated women were found to be significantly more likely to have been 'cured' of a third pregnancy. Gray et al. (2010) studied the transition to higher order births in Australia. They showed that the sex composition of previous children influenced the probability that a woman would have another child, but not the timing of births. In particular, parents of two boys were found to have had a higher probability of having a further child. The age at first birth was shown to have a positive impact on the timing and a negative impact on the quantum. Li and Chloe (1997) examined the transition to a second birth in China after the implementation of the one-child family policy in 1979. They found that the introduction of the one-child policy strongly reduced the probability of having a second birth, and significantly increased the time between the first and second births. Mc Donald and Rosina (2001) studied birth intervals in the Hutterite community. Their paper showed that the later in life a woman married, the later in life she tended to have her first child. Using a mixture cure model for recurrent events, they also showed that the death of a child did not significantly influence the probability of having an additional child, but tended to reduce the time until the next birth. Beaujouan and Solaz (2013) estimated the transition to a first child in higher order unions in a discrete time framework, where the main purpose of the model was to eliminate the quantum effect. Their main finding is that women in higher order unions speed up fertility when they advance to their biological limits of fertility. 
In all of the abovementioned studies, a parametric distribution was specified to model the time to an extra birth. In this paper, we model the transition to the next child using a flexible non-parametric model, based on Bayesian P-splines (Bremhorst and Lambert 2016). Flexible nonparametric modeling was used by demographers before: For example, Gayawan and Adebayo (2013) used Bayesian P-splines to model the baseline and the non-linear effects in an extended Cox model when studying the age at first birth in Nigeria, while calibrated splines were specified by Schmertmann (2012) to obtain a flexible estimation of the fertility schedule from abridged data. In this work, we employ post-estimation procedures to visualize the baseline intensities for the total population and the 'susceptible' women (those who had another child). Most importantly, our estimates show that the fertility schedules of the two populations differ considerably. This finding has major implications for standard event history modeling. It suggests that the shape of the baseline intensity in standard event history models is very sensitive to the size of the 'immune' population.

The paper is structured as follows: In Section 2 we provide background information on birth dynamics in Germany and summarize previous research that examined the link between education and fertility. In Section 3 we describe the methodology and the data. We use data from the German Socio-Economic Panel to investigate the transition to second and third births. As our method is unable (so far) to handle time-varying covariates, we do not study the progression to first birth. Many important covariates (such as education) may be treated as time-constant covariates in second and third birth models (they can be fixed at the start of the process), while this is not reasonable for the analysis of first births. In Section 4 we first discuss the results from the promotion time model and provide graphical representations that highlight the importance of distinguishing timing from quantum in the analysis of birth dynamics. Then a comparison with the results given by a classical Cox model is presented. In Section 5 we conclude by discussing the strengths and the limitations of the promotion time model. The main advantage of this model is that it is able to separate timing from quantum effects, while a significant limitation of the model is that its methodology is designed for time-constant covariates only. Finally, we discuss how the model could be extended to enable the inclusion of time-varying covariates.

\section{Context and previous research}

As in most other western European countries, the period fertility rate in Germany declined rapidly in the late 1960s. Since then, the period fertility rate in western Germany has been at a surprisingly stable level of around 1.4. From a cohort perspective, fertility has declined gradually over time. The western German cohort born 
in 1930 had a fertility rate of 2.1, and thus a rate that was around replacement level. For the cohorts born around 1965, this value declined to 1.5 (Pötzsch 2010; Human Fertility Database 2015). An important component of the low fertility levels in (western) Germany is the rather high share of women who remain childless. About 22 percent of (western) German women who are now approaching the end of their reproductive period will remain childless (Kreyenfeld and Konietzka 2016). It is well known that in eastern Germany the total fertility rate fell sharply after German reunification, to below one. Recently, the period birth rates in eastern and in western Germany have again reached parity (Goldstein and Kreyenfeld 2011). In addition to gathering data on total fertility, the Federal Statistical Office of Germany now also collects information on biological birth order and spacing. According to these vital statistics data, in 2010 the mean length of time between the first and the second birth was around four years in western Germany and five years in eastern Germany, while the average length of time between the second and the third birth was 4.8 years in western Germany and 5.4 years in eastern Germany (Pötzsch 2012). The vital statistics do not, however, provide information on exposure rates or on birth behavior by socioeconomic subgroup, such as by educational attainment.

Yet, as in other countries, there is a large body of literature in Germany that has examined educational differentials in birth behavior based on survey data. One of the most important studies for Germany is a paper published by Blossfeld and Huinink (1991) in the American Journal of Sociology, in which the authors used event history techniques in the analysis of first births. At that time, modeling educational participation as a time-varying covariate in fertility models was a methodological innovation. This approach allowed the authors to separate the effect of educational participation from the effect of educational attainment. The most significant innovations that followed in the field were applications that accounted for individual specific heterogeneity (Gottard, Mattei, and Vignoli 2015), models that paid attention to the selection into a field of education (Begall and Mills 2012; Oppermann 2014), and models that considered educational choice and birth dynamics as simultaneous processes (Upchurch, Lillard, and Panis 2002; Tesching 2012). Models that were able to draw upon couple data, and thus included the partner's educational characteristics in the analysis, also helped researchers gain a better understanding of the role of female education in birth progression (Bauer and Jacob 2010; Nitsche et al. 2015). An aspect of this issue that was only rarely addressed is the inability of the dependent variable in event history models to differentiate between the timing of births and ultimate parity progression. This is a serious shortcoming, particularly if the goal is to understand the effect of education on birth decisions, as there are good arguments that support the interpretation of the data in both directions: Namely, that differences in women's educational levels are linked to variations in birth timing and differences in ultimate 
parity progression. Thus, event history researchers have argued about the correct interpretation of their findings.

\section{Methods and data}

\subsection{Methods and research strategy}

\subsubsection{Cure survival models}

Cure survival models extend classical time-to-event models when an unknown fraction of the population under study never experience the event of interest; they enable the simultaneous estimation of the quantum and of the timing of the monitored event. Two main families of models can be distinguished: the mixture and the non-mixture (also referred to as the promotion time model) cure models. The mixture cure model, first introduced by Berkson and Gage (1952) and extensively studied in the statistical literature afterward (see Peng and Dear 2000; Peng 2003; Lu 2010 among others), defines the population survival function as a mixture of contributions due to the susceptible and the non-susceptible sub-populations:

$$
S_{p}(t)=(1-\pi)+\pi S_{s}(t),
$$

where $\pi$ denotes the probability of being susceptible and $S_{s}(t)$ is the survival function of the susceptible subjects. In this work we focus on the second family. The promotion time model was first motivated by the biological mechanism in the development of cancer (Yakovlev and Tsodikov 1996; Tsodikov 1998; Chen, Ibrahim, and Sinha 1999): The model assumes that each subject is exposed to $N \sim P(\theta)$ (Poisson distributed) independent latent factors (corresponding to carcinogenic cells in cancer studies) with a time to detection (of a tumor generated from a given cell) having a common proper distribution function $F(t)$. The time-to-event is defined as the minimum of the $N$ latent event times. However, its motivation can easily be translated in the realm of fertility studies. Indeed, when studying the transition to second or third birth, a latent factor could be seen as a potential decisive argument to decide to have an additional child and the 'time for its detection' as the time required for it to be convincing. It is assumed that the latent factors (or potential decisive arguments) are all defined at the onset of the process (i.e., directly after the birth of the previous child) without possibility of an evolution of their number during the follow-up of the subject. Then, the population survival function of the promotion time model can be shown to be 


$$
S_{p}(t)=\exp [-\theta F(t)]
$$

The probability of never having a second (third) child is given by:

$$
\mathrm{P}[\mathrm{N}=0]=\exp (-\theta) .
$$

The independent variables, measured at the beginning of the follow-up and denoted by $\boldsymbol{x}$ and $\boldsymbol{z}$, enter the model through a $\log$-link on parameter $\theta$ (which increases with the probability of having a second or third child) and a Cox model for the latent distribution $F(t)$ (related to the timing of an extra birth):

$$
\begin{aligned}
& \theta(\boldsymbol{x})=\exp \left(\beta_{0}+\beta_{1} x_{1}+\cdots+\beta_{p} x_{p}\right) ; \\
& F(t \mid z)=1-S_{0}(t)^{\exp \left(\lambda_{1} z_{1}+\cdots+\lambda_{q z_{q}}\right)} .
\end{aligned}
$$

As pointed out by Bremhorst and Lambert (2016), covariate vectors $\boldsymbol{x}$ and $\boldsymbol{z}$ can share some components and remain identifiable provided that the follow-up of the study is sufficiently long. In practice, if the Kaplan-Meier estimate of the survival function shows a plateau in the right tail of the distribution, then the sufficiently long follow-up assumption is respected.

In this work, the logarithm of the baseline hazard function $h_{0}(t)$, which yields the baseline survival function $S_{0}(t)$ in the Cox model, is specified using a linear combination of a large number of cubic B-splines combined with a roughness penalty on finite differences of adjacent B-spline coefficients to force smoothness (Eilers and Marx, 1996, 2010):

$$
\log \left(h_{0}(t)\right)=\sum_{k=1}^{K} \varphi_{k} b_{k}(t) \quad \text { and } \quad \tau \sum_{k}\left(\Delta^{r} \varphi_{k}\right)^{2},
$$

where $\left\{b_{k}(),. k=1, \ldots, K\right\}$ denotes the cubic B-splines basis associated with a predefined number of equidistant knots on the follow-up interval and $\tau$ is the penalty parameter. For identification purposes, the last spline coefficient is set to an arbitrarily large enough value (say, 10 ) to force $\widehat{S_{0}}($.$) to be virtually 0$ at the end of the follow-up (Bremhorst and Lambert, 2016).

The model specification provides a flexible and smooth estimation of the population hazard function $h_{p}(t)$ and of the hazard function $h_{s}(t)$ of the susceptible women. Both functions can be obtained from the population survival functions and are given by: 


$$
h_{p}(t)=\theta f(t) \quad \text { and } \quad h_{s}(t)=\frac{s_{p}(t)}{s_{p}(t)-\exp (-\theta)} h_{p}(t),
$$

where $f(t)$ is the latent density function.

\subsubsection{Bayesian inference}

Instead of working with a penalized likelihood, in the Bayesian framework the roughness penalty enters through the multivariate normal prior distribution of the spline coefficients (Lang and Brezger 2004):

$$
\varphi \sim N_{K}\left(0, \tau \varphi^{\prime} P \varphi\right),
$$

where $\mathbf{P}=\mathbf{D}^{\prime} \mathbf{D}+\varepsilon \boldsymbol{I}_{\boldsymbol{k}}$ is a full rank matrix for some small quantity $\varepsilon$ and $\mathbf{D}$ is the $\mathrm{r}^{\text {th }}$ order difference matrix. For the penalty parameter $\tau$, the robust prior distribution suggested by Jullion and Lambert (2007) is specified :

$\tau \mid \delta \sim G\left(\frac{v}{2}, \frac{v \delta}{2}\right) \quad$ and $\quad \delta \sim G\left(a_{\delta}, b_{\delta}\right)$,

where $G(a, b)$ defines a Gamma distribution with mean $\frac{a}{b}$. Because it can be shown that $v$ has a posterior distribution close to the uniform when $a_{\delta}=b_{\delta}$ are small enough (0.0001, say), fixing $v$ (equal to 2, for example) has no impact on the shape of the survival curve. Independent normal distributions with a large variance are used as priors for each regression coefficient.

The results presented in Tables 3 and 5 were obtained using MCMC with chains of length 150,000 , including a burn-in of 50,000, with parameter estimates given by the median of their posterior sample. The chains were generated using adaptive multivariate Metropolis steps for the spline and the regression coefficients (Haario, Saksman and Tamminen 2001; Atchadé and Rosenthal 2005). Gibbs steps are used to sample from the posterior of the penalty parameters $\tau$ and $\delta$ because their conditional posterior distributions belong to the Gamma family:

$$
\tau \mid \boldsymbol{\varphi}, \delta, \boldsymbol{D} \sim G\left(\frac{v+K}{2}, \frac{v \delta+\boldsymbol{\varphi}^{\prime} \boldsymbol{P} \boldsymbol{\varphi}}{2}\right) \quad \text { and } \quad \delta \mid \tau, \boldsymbol{D} \sim G\left(a_{\delta}+\frac{v}{2}, b_{\delta}+\frac{v \tau}{2}\right) .
$$

Convergence was assessed by examining the trace plots, and by using the GelmanRubin and the Geweke convergence diagnostics (Gelman and Rubin, 1992; Geweke, 
1992; Brooks and Gelman, 1998). More information on Bayesian analysis can be found e.g., in Gelman et al. (2013).

\subsection{Data}

\subsubsection{Sample}

The data for the following analyses are from the German Socio-Economic Panel (v31.0) (Wagner, Frick, and Schupp, 2007). ${ }^{5}$ The German Socio-Economic Panel (GSOEP) is a representative panel study for Germany. The first wave of this survey was launched in 1984, and the respondents have been interviewed on an annual basis since then. Over time, the GSOEP has been extended through the addition of various subsamples. Most importantly, an oversample of the eastern German population was added to the survey in 1990. Migrants and foreigners are also overrepresented. As analyzing the fertility of migrants and of eastern Germans would have required us to conduct a separate investigation, we limited our investigation to German nationals who were born in and are currently living in western Germany. ${ }^{6}$ It should be noted that individual respondents may have entered or left our study population if their location or citizenship changed.

We have also restricted our sample to female respondents, as in the GSOEP the fertility histories of women are more reliable than those of men. However, the characteristics of each woman's co-residential partner, and particularly his (or, if the woman is in a same-sex union, her) educational attainment, are accounted for in our analysis. The sample is further restricted to women who were still of childbearing age (17-49) when they entered the panel. Respondents were censored after the maximum duration of 180 months (15 years) after they had their first and their second child, or when they dropped out of the survey. Respondents who dropped out of the panel after participating in the survey for just one year were eliminated as well, as they could not contribute any exposure time to our study. Twins births were not considered in the analysis for the same reason. Cases with invalid birth histories or missing information on educational attainment were also omitted from the sample. As our focus in this study is on second and third births, we further restricted our investigation to respondents who were at risk of having a second or a third child. Left-truncated cases were also

\footnotetext{
5 The data that we used for this analysis is available in the SOEP-archive for reanalysis of published findings: https://www.diw.de/en/diw_01.c.340860.en/soep_reanalyses.html. R programs for replicating this paper's results are available upon request from the authors.

${ }^{6}$ The SOEP includes an additional high income subsample that was drawn in 2002. We also removed this subsample from the analysis, because the high income groups would otherwise be overrepresented in our data.
} 
eliminated. Thus, only respondents who had a first or a second child after they entered the panel study are used in our analysis. We also eliminated the few cases with missing information on the month of childbirth. The final sample comprises 1406 female respondents at risk of a second birth, of whom 674 reported having had a second child (before censoring). There were 1186 women at risk of having a third child, of whom 219 gave birth to a third child (before censoring).

\subsubsection{Dependent variable: transition to a second and a third child}

We have monthly information on second and third births. The process time for the second birth is the elapsed time since the birth of the first child (measured in months). The process time for the third birth is the elapsed time since the second birth. For the censored cases, the process time is the duration since the last birth until censoring.

In Figure 1, we display the probabilities of transitioning to a second and a third child. The plateau in the right tail suggests that a substantial fraction of the population will not experience the event of interest. (It represents the cure fraction, i.e., women who will never have an additional child.) As we can see in the figures, among western German women the median length of time between the first and the second birth was about four years. The parity progression ratio (when the first child was age 10) was around 65 percent. When we look at third births, we see that about 25 percent of the population at risk eventually experienced the event of interest. Thus, the 'cure fraction' for third births was about 75 percent. It should be noted that foreign nationals were excluded from the sample. As in other European countries, foreigners and migrants in Germany have higher fertility rates than the native population, and have higher progressions to the third child (Milewski 2010). Thus, the inclusion of this group would have resulted in different patterns (Kreyenfeld 2015). 
Figure 1: Transition probabilities to second and third births (Kaplan-Meier survival estimates)
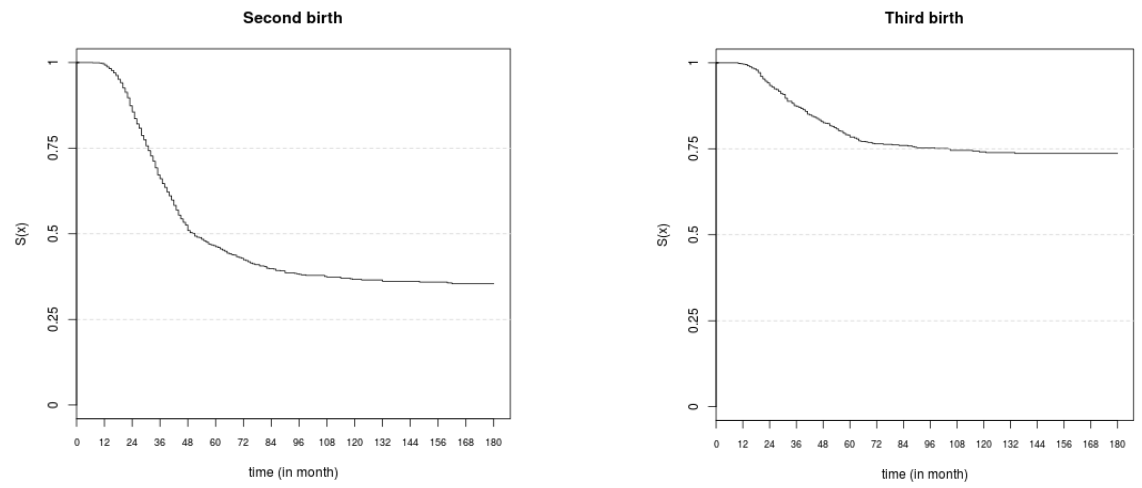

Source: SOEP 1984-2013, (v31.0), only Germans living in western Germany, high income sample was excluded.

\subsubsection{Independent variables}

Our data span the period 1984-2013. We have organized the data into person-month formats. The analyses include time-constant covariates only, which are 'frozen' at the time of the onset of the process. For example, for the analysis of the second birth, we employ information on the educational level and the partnership status at the first birth. For the third birth models, all of the covariates take the value at the birth of the second child. As we mentioned in Section 3.1, the probability of (never) having an additional child is modeled through the mean number of latent factors (cf. eq. 3). Since the promotion time model assumes that the latent factors occur only at the beginning of the study, it would not be reasonable to let the time dependent covariates influence this probability.

Our main variable of interest is educational attainment, set according to three categories: low (less than a vocational training degree), middle (a vocational training degree), and high (a university or a college degree). The respondents who had not earned a secondary degree and were still in education at the time of their first birth were also classified as having a low level of education. It should be noted that only a small fraction (less than one percent) of the respondents fell into this category at the start of the process time. We also control for partner's education using the same classification structure. There is an additional 'no partner' category for those respondents who had no partner at the time of the interview. The respondents for whom the partnership status was unclear were also assigned to this category. 
The control variables are age at the first birth and calendar period. Both are employed as a continuous covariate. For the third birth, we also controlled for the spacing of the first two births using a continuous variable that depicts the length of time between the previous two births. Tables 1 and 2 display by parity the descriptive statistics of the categorical and continuous independent variables, respectively. It should be noted that only a small fraction of women were partnered with a man who had a low level of education. This pattern may be the result of less educated men having lower chances than better educated men of entering a co-residential union (Bastin 2016).

Table 1: Descriptive statistics of the independent categorical variables for all mothers at risk of an extra birth (column 'At risk') or restricted to women who were reported to have an additional child (column 'Event')

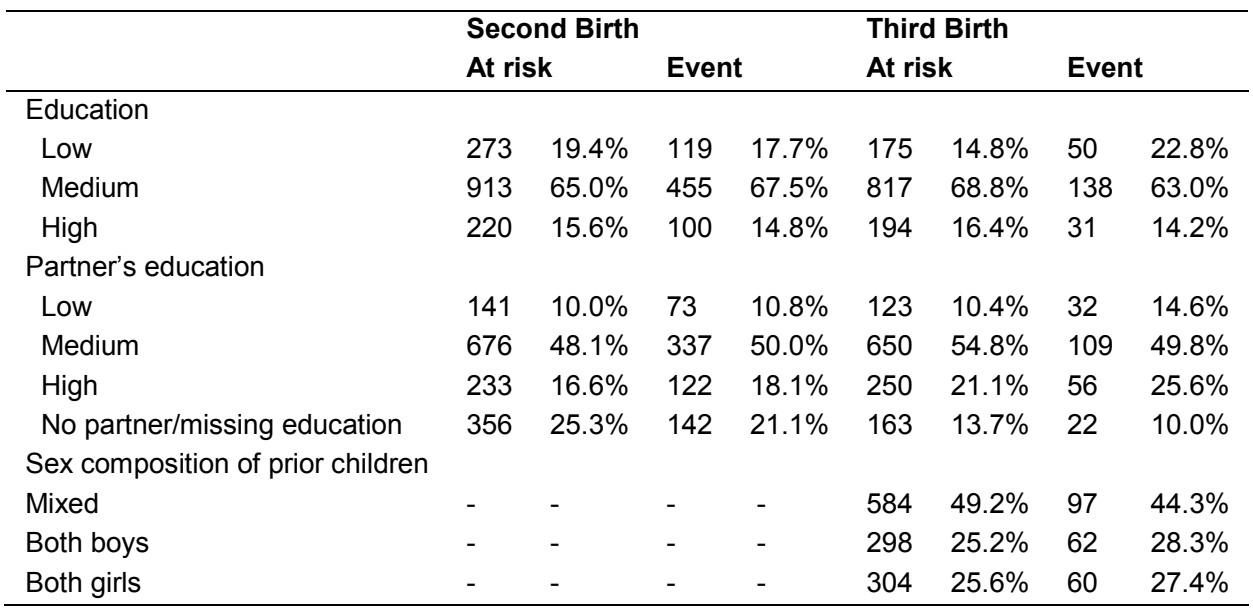

Source: SOEP 1984-2013, (v31.0), only Germans living in western Germany, high income sample was excluded. 
Table 2: Descriptive statistics of the independent continuous variables (in year) for all mothers at risk of an extra birth (column 'At risk') or restricted to women who were reported to have an additional child (column 'Event')

\begin{tabular}{llccrr}
\hline & & \multicolumn{2}{c}{ Second Birth } & \multicolumn{2}{c}{ Third Birth } \\
& & At risk & Event & At risk & Event \\
\hline \multirow{3}{*}{ Age at first birth } & Mean & 28.28 & 27.53 & 27.32 & 25.54 \\
& Medan & 28.00 & 28.00 & 27.00 & 23.00 \\
& Standard dev. & 4.89 & 4.24 & 4.56 & 4.18 \\
Birth interval $1^{\text {st }} / 2^{\text {nd }}$ births & Mean & - & - & 3.48 & 3.02 \\
& Median & - & - & 2.83 & 2.50 \\
& Standard dev. & - & - & 2.33 & 2.11 \\
Calendar Period & Mean & 1998 & 1997 & 2000 & 1996 \\
& Median & 1999 & 1996 & 2000 & 1996 \\
& Standard dev. & 8.00 & 7.00 & 8.30 & 7.37 \\
\hline
\end{tabular}

Source: SOEP 1984-2013, (v31.0), only Germans living in western Germany, high income sample was excluded.

\section{Results}

\subsection{Second births}

Table 3 shows the regression parameters for the transition to a second birth. A positive value for the estimate in the quantum column (cf. $\boldsymbol{\beta}$ in eq. 4) means that the probability of having an additional child increases when the corresponding covariate takes a positive value and the other covariates are fixed. A positive value for the estimate in the timing column (cf. $\lambda$ in eq. 5) means that the transition to a second birth tends to happen sooner for a susceptible woman (again, for a positive value of the covariate and fixed values of the others).

We find that the probability of having a second child decreased significantly with the age of the mother at her first birth. Our results further show that the older a susceptible mother was when she had her first child, the shorter the length of time between the births of her first and her second child. This evidence is very much in line with earlier findings (see e.g., Gray et al. 2010). Of particular importance for our analysis is the effect of education: Both the woman's and the partner's educational attainments had an independent and a positive effect on the probability of having a second child: i.e., a woman with a higher educational attainment was significantly more likely to have a second child. When we look at the effect of the partner's education, we see little difference between those who had low and medium educational levels. 
However, relative to having a medium educated partner, having a highly educated partner was associated with a significantly greater probability of having a second child. The high birth rates of the university graduates could indicate that a high income of both men and women is a prerequisite for having a second child. However, in a male breadwinner regime such as Germany, it comes as a surprise that women's labor market potential supports fertility transitions. An alternative explanation is a "selection argument" (Kreyenfeld 2002; Bartus et al. 2013). In a context where work and family life is incompatible, university educated women will remain childless to a greater extent than less educated women. Those who decide for a first child may be more familyprone than other women, an aspect that will accelerate their progression to a second child. Another explanation could be that we are unable to fully eliminate the strong effect of the partner's high education on fertility, because of the substantial educational homogamy that exists in western Germany.

Table 3: $\quad$ Transition to a second birth - Results from cure survival models

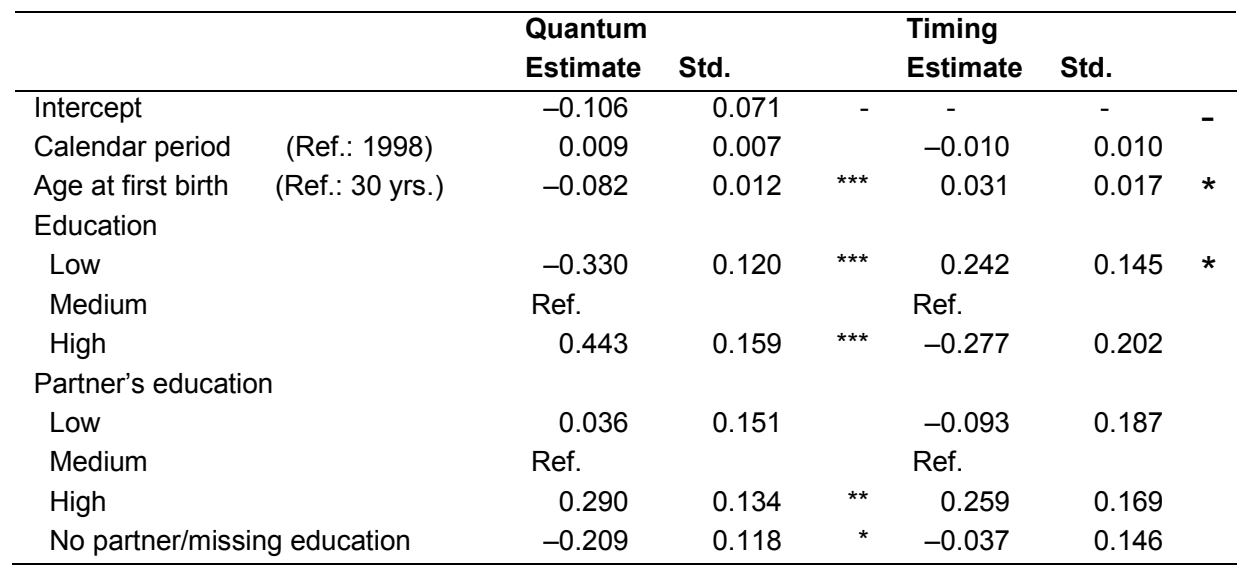

Source: SOEP 1984-2013, (v31.0), only Germans living in western Germany, high income sample was excluded. Signif. codes: ${ }^{*}=0.1 ;{ }^{* \star}=0.05 ;{ }^{* \star}=0.01$

When we look at the length of time between the births of the first two children, we see that less educated women differed significantly from other women, as they tended to have their second child sooner (for similar findings for the US, see Yamaguchi and Ferguson, 1995). We do not see any significant difference in the timing of the second child for medium and highly educated women. Neither does the partner's educational 
level have any significant influence on fertility timing. In previous research, it has been stipulated that highly educated women (and potentially also men) need to squeeze their births into a shorter period of time, because they start their childbearing career later than less educated individuals (Kreyenfeld 2002; Bartus et al. 2013). Also the argument by Ní Bhrolcháin (1986) of a "work-accelerated childbearing" would suggest that timing effects are very important for highly educated women. However, our results do not support this hypothesis.

The left-hand graph of Figure 2 shows the population hazard function (when all covariates were set at their reference values) with its 95 percent pointwise credible interval. For the whole population (women who were or were not susceptible), this function quantifies the evolution over time of the instantaneous risk of having a second child immediately after the considered time (for the women still at risk of second pregnancy). The right-hand graph of Figure 2 shows the hazard function of susceptible women (i.e., the women who will have a second child), and suggests a peak at about 3.5 years after the birth of the first child. It should be noted that as more than 90 percent of the second births occurred within 5.5 years of the first birth, the hazard functions are plotted only on this time interval (as we might otherwise be tempted to over-interpret the shape of the estimated hazard functions later on, despite the large underlying uncertainties). As expected, we find that the instantaneous risk of having a second child was higher for susceptible mothers than for the whole population. We can also see that the shapes of the two graphs differ. While the population hazard suggests that second birth intensities increased at around two to three years after the first birth, the hazard for the susceptible women peaked later.

How can we explain this difference? In the graph on the left, the immune population (those respondents who would never have a second child) are part of the risk set. At the start of the observation period, the immune population made up a 'small' fraction of the population under study. As time passed, the immune population constituted a growing fraction of the remaining risk set. Compared to a situation in which the immune population was excluded (graph on the right), the birth risks were relatively low as time passed, as the immune population remained in the sample and 'blew up' the exposure population. 
Figure 2: Transition to a second birth. The fitted population hazard function (left) and the fitted hazard function of the susceptible mothers (right) with $95 \%$ pointwise credible intervals when all covariates are set at their reference values
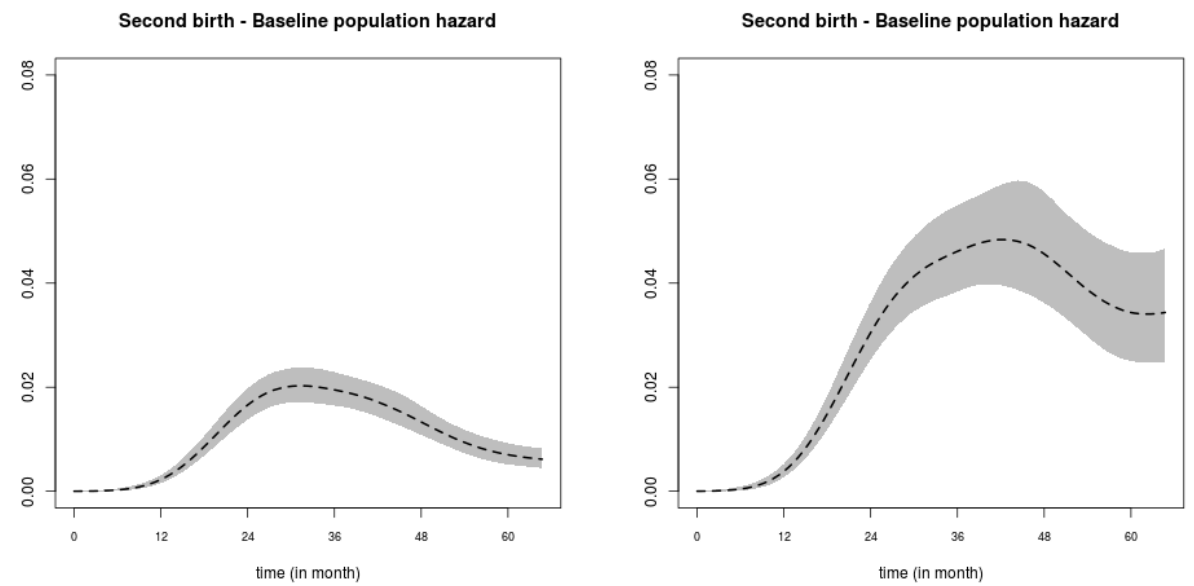

Source: SOEP 1984-2013, (v31.0), only Germans living in western Germany, high income sample was excluded.

What is the substantive meaning of the difference? Did second birth rates peak two to three years after the first birth, or did they peak later? To answer that question, let us assume for a moment that the 'immune population' are people who, for biological reasons, could not have a second child. If we accept that interpretation, the immune population should have been excluded from the representation because they were not at risk of childbearing. Thus, the figure on the right would represent the 'true' second birth profile. In reality, the people who never had a second child are a heterogeneous population, consisting, for example, of people who were unable to have another child for biological reasons, as well as of people who simply never fulfilled (for one reason or another) their desire to have another child. If we accept that interpretation, the latter group should not be removed from the risk population. It would therefore follow that the figure on the left represents the true fertility schedule. Whatever the correct assessment of the nature of the immune population, it is important to acknowledge that the fertility schedule can be strongly influenced by the inclusion or exclusion of the immune population.

Figure 3 illustrates the effects of the educational level on the hazard functions (for the reference values of the other covariates). As we saw previously in Table 3, having a 
higher educational level was associated with an increased conditional probability of having a second child (upper panel, left). Among the women who had a second child, those with the lowest educational level tended to have their second child more quickly than other women (right-hand graph). It should be noted that the suggested differences only became relevant two years after the birth of the first child. The lower row of Figure 3 illustrates the effect of the partner's educational level (for the fixed reference value of the other covariates). The left-hand graph confirms that a woman whose partner had a university degree had a greater conditional probability of having a second child (more than one year after the first birth). However, the differences suggested by the lower right-hand graph turned out to be not significant (at the 10 percent credibility level).

In a final step, we estimated the probabilities of having a child for different covariate constellations (Table 4). The most favorable configuration for having a second child (84.6 percent) was a couple in which both partners were highly educated. The groups with the lowest probability of having two children were less educated women with a medium educated partner (47.7 percent), and less educated women with a less educated partner (48.9 percent). Again we note that less educated women were especially likely to have given birth to children outside of a (residential) partnership. As we only consider the characteristics of residential partners, the fertility patterns of the less educated may not be fully captured by this table. Nevertheless, we can reject the assumption that having a higher level of education is associated with lower second birth fertility among women in Germany. Compared to medium educated women, highly educated women displayed elevated second birth probabilities, particularly when they were partnered with a highly educated man (or woman, in the few cases in which the partner was also female). 
Figure 3: Transition to a second birth. The fitted population hazard function (left), the fitted hazard function for the susceptible mothers (right). All covariates are set at their reference values, except the educational level of the mother (row 1) and of her partner (row 2)

Second birth - Population hazard function

Women educational level

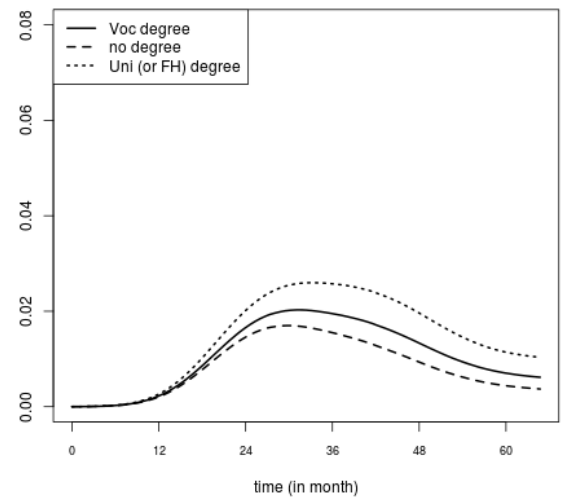

Second birth - Population hazard function

Partner educational level

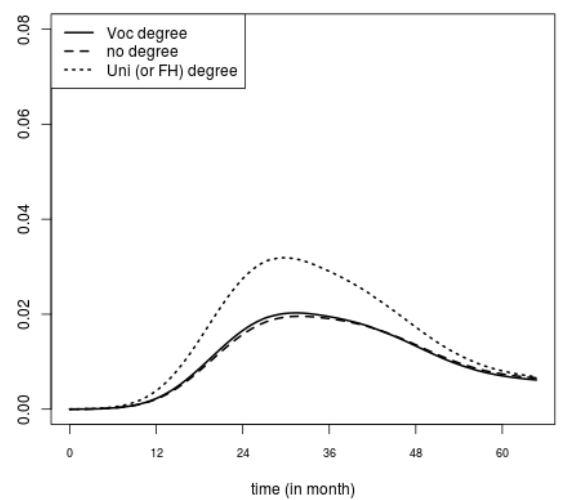

Second birth - Susceptible hazard function

Women educational level

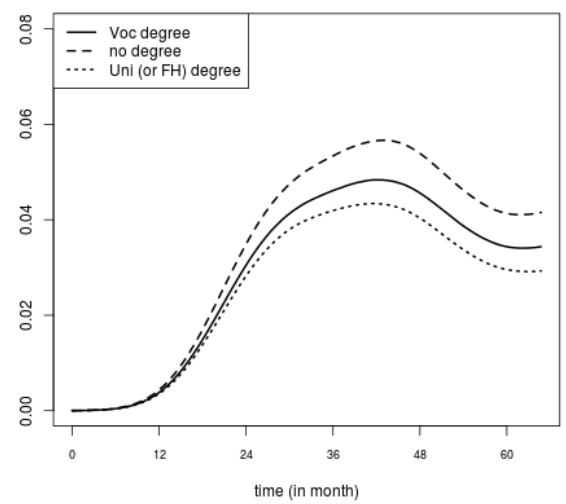

Second birth - Susceptible hazard function

Partner educational level

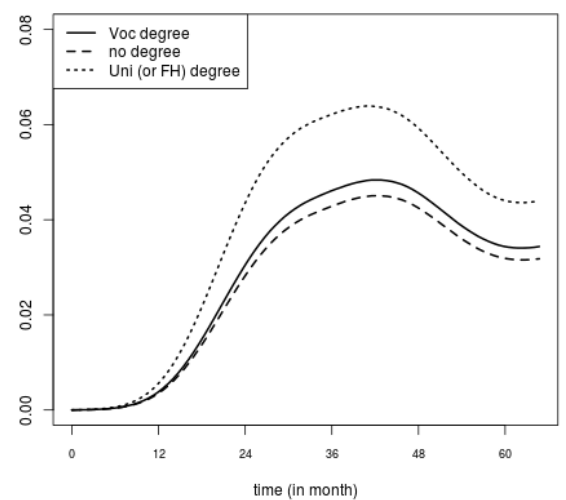

Source: SOEP 1984-2013, (v31.0), only Germans living in western Germany, high income sample was excluded. 
Table 4: Probabilities of a second birth (in \%). The estimates correspond to the posterior medians

\begin{tabular}{llll}
\hline Women's education & Partner's education & Estimate & $\mathbf{9 5 \% ~ C . I . ~}$ \\
\hline Low & Low & 48.9 & {$[37.9 ; 60.9]$} \\
Medium & Low & 60.6 & {$[50.1 ; 71.1]$} \\
High & Low & 76.6 & {$[62.5 ; 88.5]$} \\
Low & Medium & 47.7 & {$[39.2 ; 56.6]$} \\
Medium & Medium & 59.3 & {$[54.2 ; 64.5]$} \\
High & Medium & 75.3 & {$[64.6 ; 85.7]$} \\
Low & High & 57.9 & {$[46.7 ; 69.2]$} \\
Medium & High & 69.9 & {$[61.1 ; 78.1]$} \\
High & High & 84.6 & {$[74.9 ; 92.5]$} \\
\hline
\end{tabular}

Source: SOEP 1984-2013, (v31.0), only Germans living in western Germany, high income sample was excluded.

\subsection{Third births}

Table 5 reports the results for third births. Unfortunately, the sample size for the analysis of this birth order was small, which may explain why we do not find many statistically significant associations. As for the second birth, the probability of having an additional (third) child was significantly lower when the age at the first birth was high. However, the age at the first birth does not seem to have influenced the length of time between the births of the second and the third child. As might be expected, we find that the longer the time between the births of the first two children, the smaller the probability that a woman would have a third child, and the sooner the third child - if there was one - would be born. Unlike the previous studies on the topic (see e.g., Yamaguchi and Ferguson 1995; Gray et al. 2010), we do not find that the sex composition of the previous children significantly influenced the progression to a third child. It should, however, be noted that the direction of the effect was similar to the effect found in previous studies, which suggests that couples strive for a mix of genders among their children. Neither the woman's nor the partner's education had any significant influence on the timing of the next birth. However, we find that the partner's education mattered for fertility quantum: a woman with a less educated or a highly educated partner had a greater probability of having a third child than a woman with a medium educated partner. This supports earlier investigations that have shown that there is a bifurcation of fertility in western Germany, where either the disadvantaged 
groups on the labor market or the 'high earning potentials' advance to births of higher order (Kreyenfeld 2002; Kreyenfeld and Andersson 2014). To buttress that claim it would be useful to include labor market earnings into the analysis. Furthermore, there are many other confounding factors that mitigate the relationship, among them values and attitudes that strongly correlate with educational attainment. Neither can we rule out that selectivity explains some of the findings. Lower educated men are more likely to remain childless. If they father a child, they often do not move in with a partner (Bastin 2016). Because we only observe the male partner's characteristics if he lives with the respondent, we may catch a selective group of committed low educated men.

Table 5: Transition to a third birth - Results from cure survival models

\begin{tabular}{|c|c|c|c|c|c|c|}
\hline & \multicolumn{3}{|l|}{ Quantum } & \multicolumn{3}{|l|}{ Timing } \\
\hline & Est & Std & & Est & Std & \\
\hline Intercept & -1.968 & 0.156 & - & - & - & - \\
\hline Calendar period $\quad$ (Ref : 1998) & -0.004 & 0.011 & & -0.022 & 0.013 & * \\
\hline Age at first birth $\quad$ (Ref : $30 \mathrm{yrs})$ & -0.126 & 0.021 & $* * *$ & 0.016 & 0.028 & \\
\hline Birth interval $1^{\text {st }} / 2^{\text {nd }}$ births (Ref : 3 yrs) & -0.203 & 0.040 & $* * *$ & 0.081 & 0.043 & * \\
\hline \multicolumn{7}{|l|}{ Sex composition of prior children } \\
\hline Mix & Ref. & & & Ref. & & \\
\hline Both boys & 0.176 & 0.173 & & -0.034 & 0.204 & \\
\hline Both girls & 0.192 & 0.174 & & -0.228 & 0.211 & \\
\hline \multicolumn{7}{|l|}{ Education } \\
\hline Low & 0.214 & 0.190 & & -0.092 & 0.242 & \\
\hline Medium & Ref. & & & Ref. & & \\
\hline High & 0.112 & 0.239 & & 0.070 & 0.287 & \\
\hline \multicolumn{7}{|l|}{ Partner's education } \\
\hline Low & 0.521 & 0.224 & $* *$ & -0.309 & 0.302 & \\
\hline Medium & Ref. & & & Ref. & & \\
\hline High & 0.717 & 0.192 & $* * *$ & 0.349 & 0.244 & \\
\hline No partner/missing education & 0.091 & 0.253 & & 0.427 & 0.312 & \\
\hline
\end{tabular}

Source: SOEP 1984-2013, (v31.0), only Germans living in western Germany, high income sample was excluded. Signif. codes: * $=$ $0.1 ;{ }^{* *}=0.05 ;{ }^{* \star *}=0.01$ 
Figure 4 displays the hazard functions for the population (on the left) and for the susceptible mothers, i.e., women who will have a third child, (on the right) when all covariates are set at their reference values. Because only $18.5 \%$ of the sample had a third birth, the population at instantaneous risk of having a third child was, not surprisingly, rather small. If we look at the susceptible mothers only, we can see that the instantaneous risk of having a third child increased steeply during the first two years and grew more slowly thereafter. The shape of the estimated hazard function becomes more difficult to interpret over 4.5 years due to the growing uncertainty. Nevertheless, the shape of the graph shows that up to five years after the second birth, the birth hazard increased gradually. The population hazard (graph on the left) shows a rather flat pattern between ages two and five. Unfortunately, the sample sizes for third births were too small to allow us to present the graphs by level of education.

\section{Figure 4: Transition to a third birth. The fitted population hazard function (left) and the fitted hazard function of the susceptible mothers (right) with $95 \%$ pointwise credible intervals when all covariates are set at their reference values}
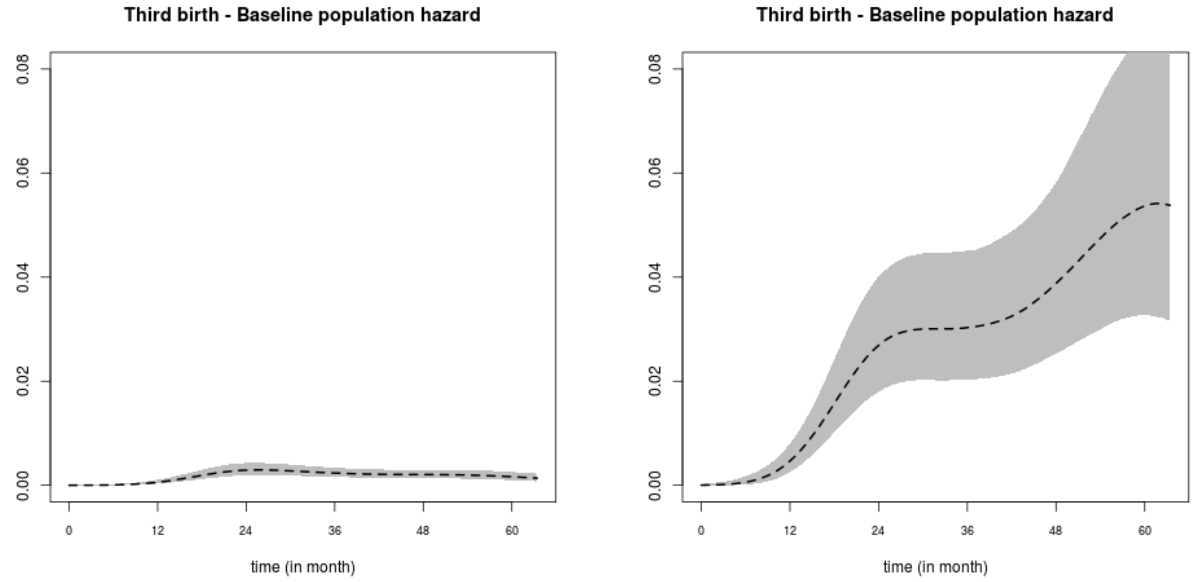

Source: SOEP 1984-2013, (v31.0), only Germans living in western Germany, high income sample was excluded.

The final step of our analysis contains the calculation of the estimated third birth probabilities for different covariate constellations (Table 6). The group with the largest (point estimation for the) probability of having a third birth was that of less educated women with a highly educated partner $(29.7 \%)$. This finding is in line with the assumption that Germany is a classical male breadwinner regime. In this regime, 
couples in which the man has the highest earning potential (measured by his high level of education) and the woman has the lowest opportunity costs of childrearing (measured by her low level of education) have the greatest chances of having a large family. This interpretation is, however, at odds with the finding that highly educated couples were also very likely to have three children. If both partners had earned a university degree, their probability of having three children was $27.4 \%$. Less educated couples had a slightly lower probability, $25.3 \%$, of having three children. Meanwhile, the medium educated couples were at a relative disadvantage: Their chances of having a third child were only around $13 \%$. Thus, they were less than half as likely to have a third child as the highly and the less educated couples.

Table 6: Probabilities of a third birth (in \%). The estimates correspond to the posterior medians

\begin{tabular}{llll}
\hline Women's education & Partner's education & Estimate & 95\% C.I. \\
\hline Low & Low & 25.3 & {$[15.2 ; 39.4]$} \\
Medium & Low & 21.0 & {$[13.3 ; 31.9]$} \\
High & Low & 23.2 & {$[13.1 ; 38.3]$} \\
Low & Medium & 15.9 & {$[10.0 ; 23.8]$} \\
Medium & Medium & 13.0 & {$[09.7 ; 17.1]$} \\
High & Medium & 14.4 & {$[08.8 ; 22.4]$} \\
Low & High & 29.7 & {$[19.0 ; 44.3]$} \\
Medium & High & 24.8 & {$[17.6 ; 34.2]$} \\
High & High & 27.4 & {$[18.5 ; 38.2]$} \\
\hline
\end{tabular}

Source: SOEP 1984-2013, (v31.0), only Germans living in western Germany, high income sample was excluded.

\subsection{Comparison with a Cox model}

One of the main assumptions of the Cox model is that all women under study will sooner or later give birth to an additional child. Looking at the Kaplan-Meier estimate of the survival function given in Figure 1, it is clear that this assumption is not supported when studying the transition to second and third births in West Germany. Nevertheless, Table 7 shows the results of a classical Cox model fitted using the coxph function of the survival package in R. These results suggest that compared to medium educated women, less educated mothers have significantly lower (conditional) second birth rates. The promotion time model teaches us that this lower value is primarily due to the smaller probability of having a second child. However, since less educated susceptible women tended to have their second child more quickly, we might have expected to see a larger ratio from a Cox model. The same conclusions can be drawn 
for the effect of age at first birth on second parity progression as well as for the effect of the time elapsed between the first two children on the transition to third birth. The Cox model results also point out that women with a low or high educated partner have a larger third birth rate compared to women with a medium educated partner. However, one cannot distinguish whether this significant effect is due to the timing or to the probability of the event while this issue is immediately highlighted by the promotion time model. This conclusion holds for high educated one child mothers compared to medium educated ones. As expected, if a factor has no significant influence on the probability and on the timing of an extra birth, the effect of this factor remains nonsignificant under the Cox model (for example, see the effect of the women's educational attainment on third parity progression).

Table 7: $\quad$ Transition to second and third births. Results from the Cox model

\begin{tabular}{|c|c|c|c|c|c|c|}
\hline & \multicolumn{2}{|c|}{ Second Births } & \multicolumn{3}{|c|}{ Third Births } & \\
\hline & LHR & Std. & & LHR & Std. & \\
\hline $\begin{array}{ll}\text { Calendar period } & \text { (Ref : 1998) }\end{array}$ & 0.005 & 0.006 & & -0.011 & 0.010 & \\
\hline Age at first birth (Ref : 30 yrs) & -0.073 & 0.010 & $* * *$ & -0.119 & 0.019 & *** \\
\hline Birth interval $1^{\text {st }} 2^{\text {nd }}$ child (Ref : $3 \mathrm{yrs}$ ) & - & - & - & -0.183 & 0.039 & *** \\
\hline \multicolumn{7}{|l|}{ Sex composition of prior children } \\
\hline Mix & - & - & - & Ref. & & \\
\hline Both boys & - & - & - & 0.153 & 0.164 & \\
\hline Both girls & - & - & - & 0.139 & 0.166 & \\
\hline \multicolumn{7}{|l|}{ Education } \\
\hline Low & -0.240 & 0.112 & ** & 0.171 & 0.182 & \\
\hline Medium & Ref. & & & Ref. & & \\
\hline High & 0.336 & 0.123 & $* * *$ & 0.133 & 0.228 & \\
\hline \multicolumn{7}{|l|}{ Partner's education } \\
\hline Low & 0.001 & 0.131 & & 0.423 & 0.212 & $* *$ \\
\hline Medium & Ref. & & & Ref. & & \\
\hline High & 0.399 & 0.116 & *** & 0.872 & 0.188 & *** \\
\hline No partner/missing education & -0.244 & 0.105 & ** & 0.216 & 0.238 & \\
\hline
\end{tabular}

Source: SOEP 1984-2013, (v31.0), only Germans living in western Germany, high income sample was excluded. Signif. codes : ${ }^{\star}=0.1 ;{ }^{\star \star}=0.05 ;{ }^{\star \star \star}=0.01$

\section{Discussion}

In this paper, transitions to second and third births were analyzed using the promotion time model (also known as the cure survival or the split-population model). This family 
of models enabled us to distinguish the factors that influence the probability of having an additional child from those that influence the timing of an additional birth. We went beyond standard cure survival models by specifying a flexible non-parametric model (using Bayesian P-splines) for the specification of the baseline intensity. Our main factor of interest was the influence of female and male education on parity progression. The results of our investigation show that the ultimate probability of having a second child increased with the level of education of the mother, but also that less educated women had their second child sooner than their better educated counterparts. When we looked at third births, we found that a woman with a highly educated partner was more likely to have had an additional child. Neither female nor male educations levels were, however, shown to have a significant influence on the spacing of the third birth.

We have also demonstrated that the shape of the fertility schedule differs depending on whether we examine the entire population or only the 'susceptible women' (those who eventually experience the event). For second births, for example, the 'full population hazard' suggests that birth intensities peak around three years after the first birth. If we focus on the susceptible women, the peak shifts by one year. While this change in the fertility schedule may be obvious for bio-statisticians, for applied researchers it has major implications for the correct interpretation of the baseline intensities of an event history model. The age (or duration) when birth intensities peak is commonly interpreted as being the point in time when it is most likely that a woman will have a child. The validity of this interpretation is arguable when the population considered at risk includes women who will never experience the event of interest. Indeed, as the share of this immune population grows over time, the peak of the fertility schedule shifts, even if the population who experience the event of interest does not change its behavior.

It is widely known in the research community that standard event history models conflate timing and quantum effects. Hence, it is surprising to observe that alternative model specifications that try to separate out the two have still not seeped into applied fertility research. An illustration of possible misleading conclusions from the classical time-to-event model was presented in Section 4.3 by comparing the results obtained from the Cox and from the promotion time models.

At the same time, however, we must acknowledge that our approach, which is able to separate the timing and the quantum of fertility, leaves an important issue unresolved. Unfortunately, in the promotion time cure model, only time-constant covariates can be used to model the probability of being susceptible. Chi and Ibrahim (2006) proposed an extension to such covariates by allowing the latent factors to occur at any time during the follow-up. We are currently working on an extension of our flexible model to handle time-varying covariates. 


\section{Acknowledgments}

The authors acknowledge financial support from IAP research network P7/06 of the Belgian Government (Belgian Science Policy), and from the contract "Projet d'Actions de Recherche Concertées" (ARC) 11/16-039 of the "Communauté française de Belgique", granted by the Académie universitaire Louvain. The authors also thank Jutta Gampe (Max Planck Institute for Demographic Research, Rostock), who inspired our collaboration. For valuable comments on an earlier draft of this paper, we would like to thank Frans Willekens (Max Planck Institute for Demographic Research, Rostock). For language editing, we are grateful to Lena Klein and Miriam Hils. The authors also thank the editor and the anonymous referees for their constructive comments and suggestions for improving this manuscript. 


\section{References}

Alter, G., Oris, M., and Tyurin, L. (2007). The shape of a fertility transition and analysis of birth intervals in Eastern Belgium. Paper presented to the Population Association of America meeting in New York.

Atchadé, Y.F. and Rosenthal, J.S. (2005). On adaptive Markov chain Monte Carlo algorithms. Bernoulli 11(5): 815-828. doi:10.3150/bj/1130077595.

Bastin, S. (2016). Partnerschaftsverläufe alleinerziehender Mütter: Eine quantitative Untersuchung auf Basis des Beziehungs- und Familienpanels. Wiesbaden: Springer VS. doi:10.1007/978-3-658-10685-0.

Bartus, T., Murinkó, L., Szalma, I., and Szél, B. (2013). The effect of education on second births in Hungary: A test of the time-squeeze, self-selection, and partnereffect hypotheses. Demographic Research 28(1): 1-32. doi:10.4054/DemRes.2013.28.1.

Bauer, G. and Jacob, M. (2010). Fertilitätsentscheidungen im Partnerschaftskontext: Eine Analyse der Bedeutung der Bildungskonstellation von Paaren für die Familiengründung anhand des Mikrozensus 1996-2004 [Fertility decisons in the partnership context: An analysis of the educational constellations of couples for family formation based on the microcensus 1996-2004]. Kölner Zeitschrift für Soziologie und Sozialpsychologie 62: 31-60. doi:10.1007/s11577-010-0089-y.

Beaujouan E. and Solaz A. (2013). Racing against the biological clock? Childbearing and sterility among men and women in second unions in France. European Journal of Population 29(1): 39-67. doi:10.1007/s10680-012-9271-4.

Begall, K. and Mills, M.C. (2012). The influence of educational field, occupation, and occupational sex segregation on fertility in the Netherlands. European Sociological Review 28(4): 1-23. doi:10.1093/esr/jcs051.

Berinde, D. (1999). Pathways to a third child in Sweden. European Journal of Population 15: 349-378. doi:10.1023/A:1006287630064.

Berkson, J. and Gage, R. (1952). Survival curve for cancer patients following treatment. Journal of the Americal Statistical Association 47: 501-515. doi:10.1080/016 21459.1952.10501187.

Blossfeld, H.-P. and Huinink, J. (1991). Human capital investments or norms of role transition? How women's schooling and career affect the process of family formation. American Journal of Sociology 97(1): 143-168. doi:10.1086/229743. 
Bremhorst, V. and Lambert, P. (2016). Flexible estimation in cure survival models using Bayesian P-splines. Computational Statistics and Data Analysis 93: 270284. doi:10.1016/j.csda.2014.05.009.

Brooks, S. and Gelman, A. (1998). General methods for monitoring convergence of iterative simulations. Journal of Computational and Graphical Statistics 7: 434455 .

Chen, M.-H., Ibrahim, J.G., and Sinha, D. (1999). A new bayesian model for survival data with a surviving fraction. Journal of the American Statistical Association 94: 909-919. doi:10.1080/01621459.1999.10474196.

Chi, Y. and Ibrahim, J. G. (2006). Joint models for multivariate longitudinal and multivariate survival data. Biometrics 62(2): 432-445. doi:10.1111/j.15410420.2005.00448.x.

Eilers, P. and Marx, B. (1996). Flexible smoothing with B-splines and penalties (with discussion). Statistical Modeling 11(2): 89-121.

Eilers, P. and Marx, B. (2010). Splines, knots, and penalties. Wiley Interdisciplinary Reviews: Computational Statistics 2(6): 637-653. doi:10.1002/wics.125.

Gayawan, E. and Samson B.A. (2013). A Bayesian semiparametric multilevel survival modelling of age at first birth in Nigeria. Demographic Research 28(45): 13391372. doi:10.4054/DemRes.2013.28.45.

Gelman, A. and Rubin, D. (1992). Inference from iterative simulation using multiple sequences. Statistical Science 7(4): 457-511. doi:10.1214/ss/1177011136.

Gelman, A., Carlin, J.B., Stern, H.S., Dunson, D.B., Vehtari, A., and Rubin, D. (2013). Bayesian data analysis. $3^{\text {rd }}$ ed. Boca Raton: Chapman and Hall/CRC.

Gerster, M., Keiding, N., Knudsen, L.B., and Strandberg-Larsen, K. (2007). Education and second birth rates in Denmark 1981-1994. Demographic Research 17(8): 181-210. doi:10.4054/DemRes.2007.17.8.

Geweke, J. (1992). Evaluating the accuracy of sampling-based approaches to calculating posterior moments. In: Bernardo, J., Berger, J., David, A., and Smith, A. (eds.). Bayesian statistics 4. Oxford: Clarendon: 169-193.

Goldstein, J.R. and Kreyenfeld, M. (2011). Has East Germany overtaken West Germany? Recent trends in order-specific fertility. Population and Development Review 37(3): 453-472. doi:10.1111/j.1728-4457.2011.00430.x. 
Gottard, A., Mattei, A., and Vignoli, D. (2015). The relationship between education and fertility in the presence of a time varying frailty component. Journal of the Royal Statistical Society, Series A 178(4): 863-881. doi:10.1111/rssa.12097.

Gray, E., Evans, A., Anderson, J., and Kippen, R. (2010). Using split-population models to examine predictors of the probability and timing of parity progression. European Journal of Population 26(3): 275-295. doi:10.1007/s10680-009-92012.

Haario, H., Saksman, E. and Tamminen, J. (2001). An adaptive Metropolis algorithm. Bernoulli 7(2): 223-242. doi:10.2307/3318737.

Hoem, J.M. (1993). Classical demographic methods of analysis and modern eventhistory techniques. Stockholm Research Reports in Demography 75. Stockholm: Stockholm University.

Huinink, J. (1989). Das zweite Kind: Sind wir auf dem Weg zur Ein-Kind-Familie? [The second child: Are we on the path towards the one-child-family?]. Zeitschrift für Soziologie 18(3): 192-207.

Human Fertility Database. (2015). Cohort fertility: West Germany. Max Planck Institute for Demographic Research (Germany) and Vienna Institute of Demography (Austria). Available at www.humanfertility.org (data downloaded on July, 16 2015).

Jullion, A. and Lambert, P. (2007). Robust specification of the roughness penalty prior distribution in spacially adaptive Bayesian P-splines models. Computational Statistics and Data Analysis 51(5): 2542-2558. doi:10.1016/j.csda.2006.09.027.

Kravdal, Ø. (2001). The high fertility of college educated women in Norway: An artefact of the separate modelling of each parity transition. Demographic Research 5(6): 187-215. doi:10.4054/DemRes.2001.5.6.

Kreyenfeld, M. (2002). Time squeeze, partner effect or self-selection? An investigation into the positive effect of women's education on second birth risks in West Germany. Demographic Research 7(2): 15-48. doi:10.4054/DemRes.2002.7.2.

Kreyenfeld, M. and Andersson, G. (2014). Socio-economic differences in the unemployment and fertility nexus in cross-national comparison. Advances in Life Course Research 21: 59-73. doi:10.1016/j.alcr.2014.01.007.

Kreyenfeld, M. (2015). Economic uncertainty and fertility. Zeitschrift für Soziologie und Sozialpsychologie 67: 59-80. doi:10.1007/s11577-015-0325-6. 
Kreyenfeld, M. and Konietzka, D. (2016). Childlessness in Germany. In: Kreyenfeld, M. and Konietzka, D. (eds.). Childlessness in Europe: Context, causes and consequences. Wiesbaden: Springer.

Lang, S. and Brezger, A. (2004). Bayesian P-splines. Journal of Computational and Graphical Statistics 13(1): 183-212. doi:10.1198/1061860043010.

Li, L. and Chloe, M. (1997). A mixture model for duration data: Analysis of second births in China. Demography 34(2): 189-197. doi:10.2307/2061698.

Lu, W. (2010). Efficient estimation for an accelerate failure time model with a cure fraction. Statistica Sinica 20: 661-674.

McDonald, J.W. and Rosina, A. (2001). Mixture modelling of recurrent event times with long-term survivors: Analysis of Hutterite birth intervals. Statistical Methods and Applilcations 10(1): 257-272.

Milewski, N. (2010). Fertility of immigrants: A two-generational approach in Germany. Berlin: Springer. doi:10.1007/978-3-642-03705-4.

Ní Bhrolcháin, M. (1986). Women's paid work and the timing of births: Longitudinal evidence. European Journal of Population 2(1): 43-70. doi:10.1007/BF0 1796880.

Ní Bhrolcháin, M. (2011). Tempo and the TFR. Demography 48(3): 841-861. doi:10.1007/s13524-011-0033-4.

Nitsche, N., Matysiak, A., Van Bavel, J., and Vignoli, D. (2015). Partners' educational pairings and fertility across Europe. Families and Societies working paper 2015/38. Stockholm: Families and Societies/University of Stockholm.

Oláh, L.S. (2003). Gendering fertility: Second births in Sweden and Hungary. Population Research and Policy Review 22(2): 171-200. doi:10.1023/A:1025 089031871.

Oppermann, A. (2014). Exploring the relationship between educational field and transition to parenthood: An analysis of women and men in Western Germany. European Sociological Review 30(6): 728-749. doi:10.1093/esr/jcu070.

Peng, Y. (2003). Estimating baseline distribution in proportional hazards cure models. Computional Statistics and Data Analysis 42(1-2): 187-201. doi:10.1016/S0 167-9473(02)00158-5.

Peng, Y. and Dear, K. (2000). A nonparametric mixture model for cure rate estimation. Biometrics 56(1): 237-243. doi:10.1111/j.0006-341X.2000.00237.x. 
Pötzsch, O. (2010). Cohort fertility: A comparison of the results of the official birth statistics and of the microcensus survey 2008. Comparative Population Studies 35(1): 185-204.

Pötzsch, O. (2012). Geburtenfolge und Geburtenabstand: Neue Daten und Befunde [Birth order and birth spacing: New data and new evidence]. Wirtschaft und Statistik 2: 89-101.

Prskawetz, A. and Zagaglia, B. (2005). Second births in Austria. Vienna Yearbook of Population Research 3: 143-170.

Rizopoulos, D. (2012). Joint models for longitudinal and time-to-event data with applications in R. Boca Raton: Chapman and Hall/CRC. doi:10.1201/b12208.

Schmertmann, C.P. (2012). Calibrated spline estimation of detailed fertility schedules from abridged data. MPIDR Working Paper 2012-022. Rostock: Max Planck Institute for Demographic Research.

Tesching, K. (2012). Education and fertility: Dynamic interrelations between women's educational level, educational field and fertility in Sweden. [PhD thesis]. Stockholm: Stockholm University. http://www.demogr.mpg.de/publications/ files/4565_1334049671_1_Full\%20Text.pdf.

Tsodikov, A.D. (1998). A proportional hazard model taking account of long-term survivors. Biometrics 54(4): 1508-1516. doi:10.2307/2533675.

Upchurch, D.M., Lillard, L.A., and Panis, C.W.A. (2002). Nonmarital childbearing: Influences of education, marriage, and fertility. Demography 39(2): 311-329. doi:10.1353/dem.2002.0020.

Wagner, G.G., Frick, J.R., and Schupp, J. (2007). The German Socio-Economic Panel Study (SOEP): Scope, evolution and enhancements. Schmollers Jahrbuch 127: 139-169. doi:10.2139/ssrn.1028709.

Yamaguchi, K. and Ferguson, L. (1995). The stopping and spacing of childbirths and their birth-history predictors: Rational choice theory and event history analysis. American Sociological Review 60(2): 272-298. doi:10.2307/2096387.

Yakovlev, A. and Tsodikov, A. (1996). Stochastic models for tumor of latency and their biostatistical applications. Hackensack: World Scientific. 